\title{
Overview of anisotropic flow measurements from ALICE
}

You Zhou on behalf of the ALICE Collaboration

Niels Bohr Institute, University of Copenhagen, Denmark

\begin{abstract}
Anisotropic flow is an important observable to study the properties of the hot and dense matter, the Quark Gluon Plasma (QGP), created in heavy-ion collisions. Measurements of anisotropic flow for inclusive and identified charged hadrons are reported in $\mathrm{Pb}-\mathrm{Pb}, \mathrm{p}-\mathrm{Pb}$ and $\mathrm{pp}$ collisions with the ALICE detector. The comparison of experimental measurements to various theoretical calculations are also presented in these proceedings.
\end{abstract}

\section{Introduction}

The study of anisotropic flow in relativistic heavy collisions at RHIC suggested that the produced system is described as a strongly coupled quarkgluon plasma (sQGP) [1]. Recent anisotropic flow measurements at the LHC indicate that the system created in $\mathrm{Pb}-\mathrm{Pb}$ collisions at $\sqrt{s_{\mathrm{NN}}}=2.76$ $\mathrm{TeV}$ also behaves as a strongly interacting liquid [2]. Meanwhile, additional constraints on the value of shear viscosity over entropy density ratio $\eta / s$ are obtained by studying the anisotropic flow of identified particles. The most pronounced component of anisotropic flow is second order harmonic, called elliptic flow $v_{2}$. In these proceedings, we will discuss the elliptic flow of both charged particles and identified particles measured in ALICE. 


\section{Anisotropic flow measurements in $\mathrm{Pb}-\mathrm{Pb}$ collisions}

The centrality dependence of elliptic flow $v_{2}$, measured with different methods, is shown in Fig. 1 (left). The results fall into two groups: (a) results from two-particle correlations have high values; $(2)$ results from multi-particle correlations have smaller $v_{2}$. The clear difference between the two groups was observed for all centrality classes. This could be due to the flow fluctuations, as well as due to the so-called non-flow effects, which is the azimuthal correlation not associate to the common symmetry planes. In addition, the $v_{2}$ measurements with the LYZ method at STAR are smaller than those at ALICE, at the level of $30 \%$. This $30 \%$ difference is bigger than the ideal hydrodynamic predictions [3] but agrees with viscous hydrodynamics calculations [4].
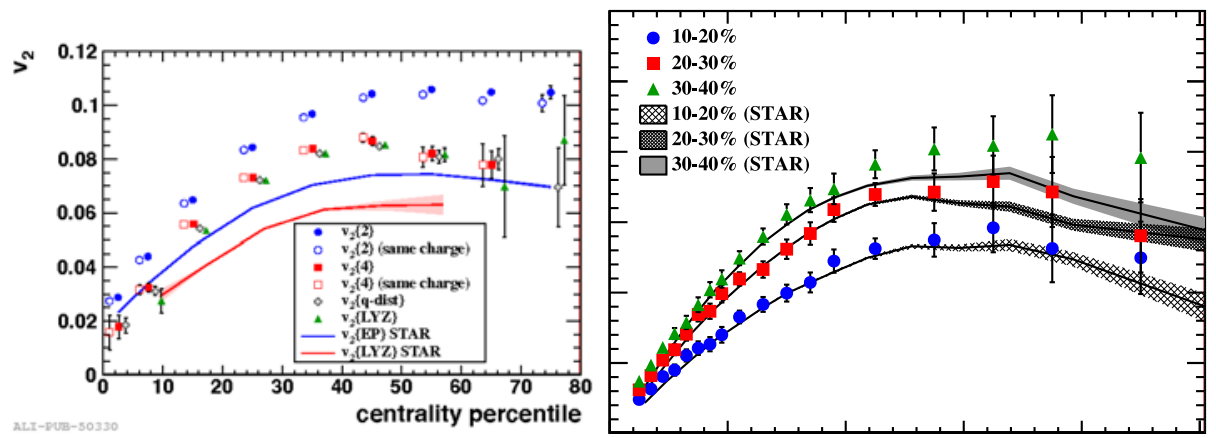

Figure 1: Centrality dependence of elliptic flow $v_{2}$ measured with various methods for RHIC and the LHC; $p_{\mathrm{T}}$-differential $v_{2}\{4\}$ of charged particles for RHIC and the LHC (right).

In order to understand the $30 \%$ increasing of integrated $v_{2}$ from RHIC to the LHC, we study the $v_{2}$ in more details with the $p_{\mathrm{T}}$ differential flow $v_{2}\left(p_{\mathrm{T}}\right)$ measurements. Figure 1 (right) shows the $v_{2}\left(p_{\mathrm{T}}\right)$ for the centrality class 10$20 \%, 20-30 \%$ and $30-40 \%$, using a 4-particle cumulant method, denoted as $v_{2}\{4\}$. The comparison for the same measurements in STAR are presented in the same centrality class. It is found that the differential $v_{2}\{4\}$ measurement does not change within uncertainties from top RHIC to LHC energies. It suggests that a large fraction of the $30 \%$ increasing integrated $v_{2}$ is due to an increase in the $\left\langle p_{\mathrm{T}}\right\rangle$.

In addition, the $v_{2}\left(p_{\mathrm{T}}\right)$ is also measured for identified particles in $\mathrm{Pb}-\mathrm{Pb}$ collisions at $\sqrt{s_{\mathrm{NN}}}=2.76 \mathrm{TeV}$ [8]. It was reported that for the low $p_{\mathrm{T}}$ region (i.e. $\left.p_{\mathrm{T}} \leq 3 \mathrm{GeV} / c\right)$, one can see a mass ordering feature $\left(v_{2}^{\pi}<v_{2}^{\mathrm{K}}<v_{2}^{\mathrm{p}} \leq\right.$ 

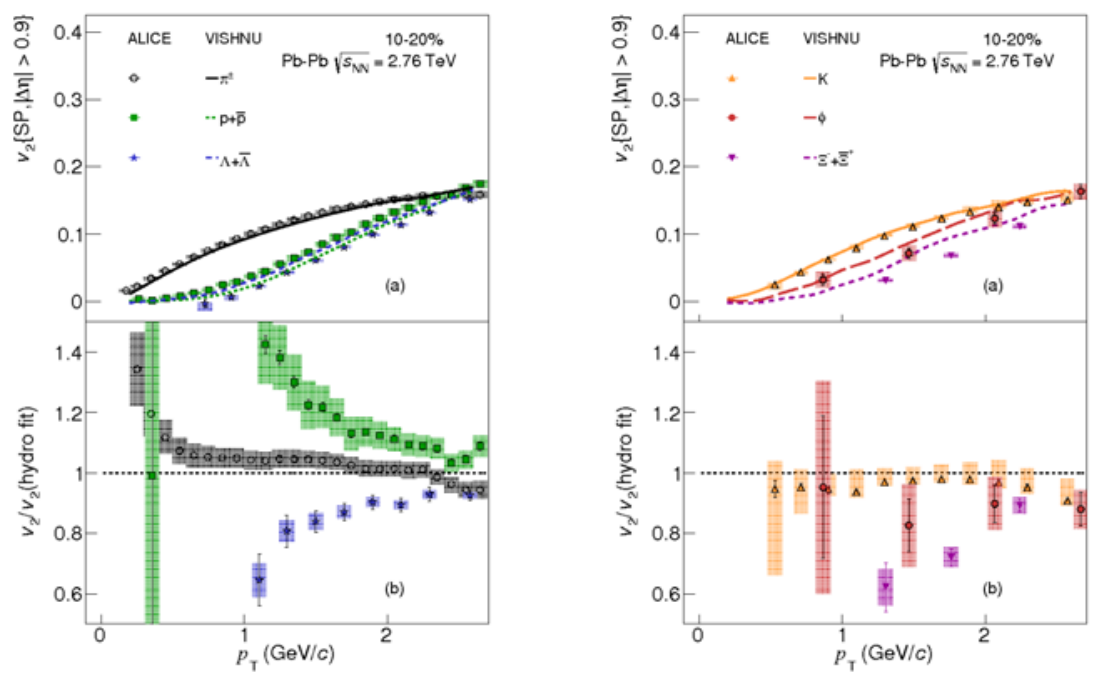

Figure 2: Measurements of dentified particle $v_{2}$ and the comparisons to the VISHNU calculations.

$\left.v_{2}^{\phi}<v_{2}^{\Lambda}<v_{2}^{\Xi}<v_{2}^{\Omega}\right)$. This is expected due to the interplay between elliptic and radial flow $[5,6]$. For higher values of $p_{\mathrm{T}}$ (i.e. $p_{\mathrm{T}}>3 \mathrm{GeV} / c$ ), particles tend to group according to their type, i.e. mesons and baryons.

Hydrodynamic calculations reveal the fact that the information of $\eta / s$ of the QGP, the hadronic chemical compositions as well as off-equilibrium kinetics can be extracted from the comparison of identified particle $v_{2}$ measurements. Figure 2 shows a comparison between data and the calculations from VISHNU [9], which is based on initial conditions modeled by MC-KLN and a shear viscosity over entropy density ratio $\eta / s=0.16$. It was found that the VISHNU calculations generate the main feature of the $v_{2}$ mass ordering like data at low $p_{\mathrm{T}}$. However, a closer look reveals that VISHNU calculations of $\pi^{ \pm}$and $\mathrm{p}(\overline{\mathrm{p}}) v_{2}$ are systematically below the measured $v_{2}$ from ALICE, while the calculations of $\mathrm{K}^{ \pm} v_{2}$ are compatible with the data. Also $v_{2}$ of heavier particles, including $\phi$ meson, strange and multi-strange particles, are overestimated in this hybrid hydrodynamic calculations compared to data. Furthermore, it is noticed that in the VISHNU calculations, the results of $\phi$ and $\Lambda(\bar{\Lambda}) v_{2}$ do not follow the precise mass ordering at low $p_{\mathrm{T}}$ region. All these results suggest that $\phi$ meson, strange and multi-strange particles might either freeze-out later than what the current implementation of VISHNU expects, and/or the hadronic cross sections of these parti- 

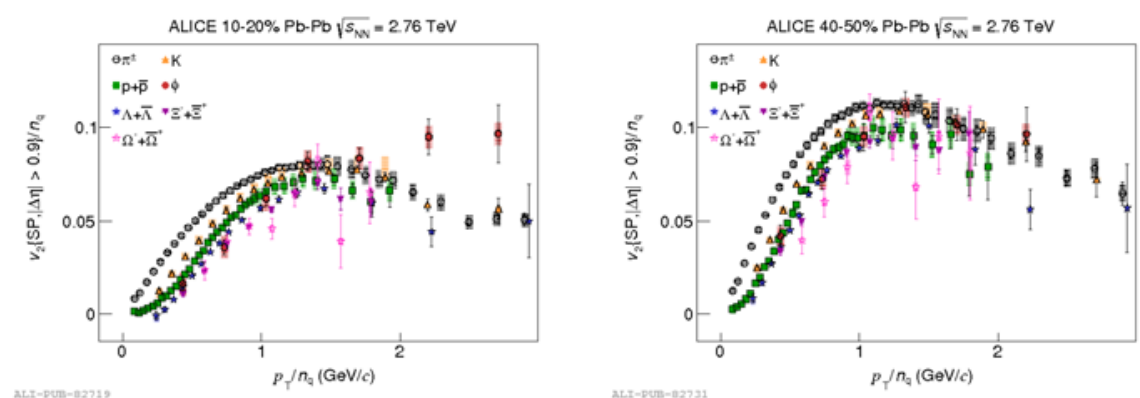

Figure 3: The $p_{\mathrm{T}} / n_{q}$ dependence of $v_{2} / n_{q}$ of identified particles in the 10-20\% and 40-50\% centrality intervals of $\mathrm{Pb}-\mathrm{Pb}$ collisions at $\sqrt{s_{\mathrm{NN}}}=2.76 \mathrm{TeV}$.

cles are underestimated in UrQMD model. Thus, further knowledge about freeze-out conditions, including the radial flow, freeze-out temperature, and better constraints of the hadronic cross section of the $\phi$ meson, strange and multi-strange particles are still necessary to improve the hydrodynamic calculations.

The number of constituent quarks scaling (NCQ scaling), was used to support the picture that the partonic collectivity already develops at top RHIC energy [7]. It serves as an examination of hadron production via the quark coalescence/recombination mechanism. The $v_{2}$ scaled by the number of constituent quarks $\left(n_{q}\right)$ as a function of $p_{\mathrm{T}} / n_{q}$ for all particle species are shown in Fig. 3. It is clear that the NCQ scaling does not hold perfectly, the deviations of this scaling from unity are at the level of $\pm 20 \%$ for all centrality classes.

\section{Anisotropic flow measurements in $\mathrm{p}-\mathrm{Pb}$ and $\mathrm{pp}$ collisions}

The measurements of anisotropic flow in $\mathrm{p}-\mathrm{Pb}$ collisions at the LHC were originally aimed to provide reference data for the $\mathrm{Pb}-\mathrm{Pb}$ collisions, e.g. study the cold nuclear matter effects. However, unexpected collective behaviors have been discovered in experiments. Figure 4 show the two- and four-particle cumulants as a function of the charged particle multiplicity in $\sqrt{s_{\mathrm{NN}}}=5.02 \mathrm{TeV} \mathrm{p}-\mathrm{Pb}$ collisions. It was observed that the two-particle cumulant decreases with increasing charged particle multiplicity. Various pseudorapidity gaps between the two particles have been examined. It was realized that using a pseudorapidity gap $|\Delta \eta|>1.4$, the two-particle cumulant is 

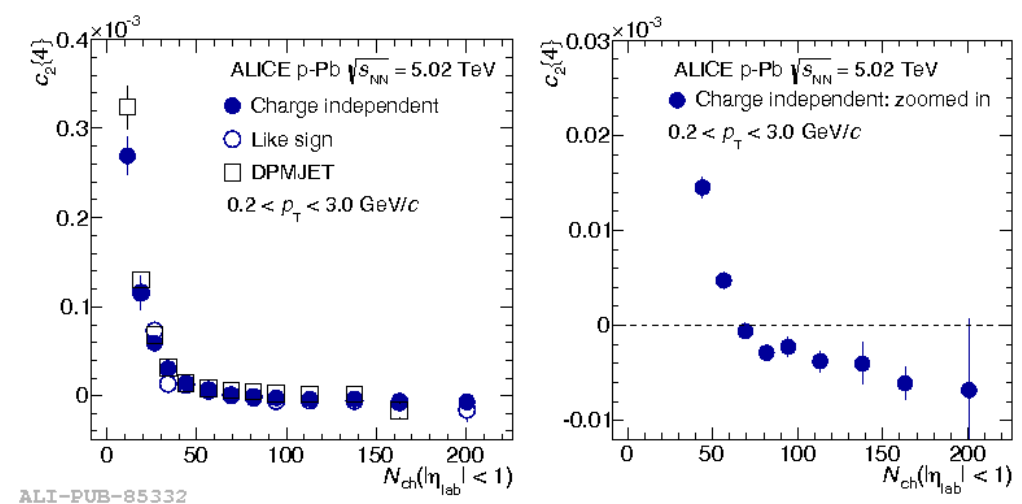

Figure 4: Two- and multi-particle cumulant as a function of the charged particle multiplicity in $\mathrm{p}-\mathrm{Pb}$ collisions.
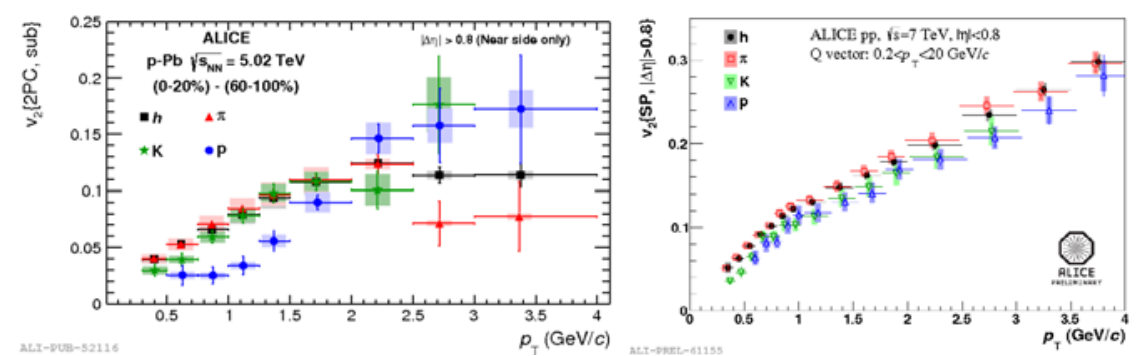

Figure 5: $p_{\mathrm{T}}$-differential $v_{2}$ for all charged and identified particles in $\mathrm{p}-\mathrm{Pb}$ collisions for the $0-20 \%$ multiplicity class (left) and in minimum biased pp collisions (right).

strongly suppressed, and an increase with increasing multiplicity is observed. In addition, the four-particle cumulant, which is less sensitive to the nonflow effects, was measured for the same multiplicity region. It was observed that for the events with a multiplicity above 70, the four-particle cumulant becomes negative, which is a prerequisite to extract $v_{2}\{4\}$, due to $v_{2}\{4\}=$ $\sqrt[4]{-c_{2}\{4\}}$. Similar analysis was also performed in pp collisions at $7 \mathrm{TeV}[11]$, no negative sign of $c_{2}\{4\}$ has been observed. The anisotropic flow signal in pp collisions, has not been observed for the presented multiplicity range.

The identified particle $v_{2}$ as a function of $p_{\mathrm{T}}$ was also investigated in $\mathrm{p}-\mathrm{Pb}$ collisions. This result was obtained using a two-particle correlation technique. It was shown that after applying the subtraction procedure using 60-100\% multiplicity events to remove the non-flow contributions, a mass ordering is observed, which is in qualitatively agreement with hydrodynamic calculations. It was noticed that the mass ordering feature can be also re- 
produced in UrQMD simulations in p- $\mathrm{Pb}$ collisions. This is the consequence of hadronic interactions [12]. Figure 5 (right) shows the identified particle $v_{2}$ measurements in minimum bias pp collisions at $\sqrt{s}=7 \mathrm{TeV}$. It seems that the $v_{2}$ of pion is consistent with that of kaon for $p_{\mathrm{T}}<3 \mathrm{GeV} / c$, while it is higher than $v_{2}$ of proton for the entire $p_{\mathrm{T}}$ range. This behavior is different from the mass ordering feature observed in both $\mathrm{Pb}-\mathrm{Pb}$ and $\mathrm{p}-\mathrm{Pb}$ collisions, presented in Fig. 2 and 5 (right).

\section{References}

[1] I. Arsene et al. [BRAHMS Collaboration], Nucl. Phys. A 757, 1 (2005); K. Adcox et al. [PHENIX Collaboration], Nucl. Phys. A 757, 184 (2005); J. Adams et al. [STAR Collaboration], Nucl. Phys. A 757 (2005) 102

[2] K. Aamodt et al. [ALICE Collaboration], Phys. Rev. Lett. 105, 252302 (2010); Phys. Rev. Lett. 107, 032301 (2011)

[3] G. Kestin and U. W. Heinz, Eur. Phys. J. C 61, 545 (2009)

[4] H. Niemi, K. J. Eskola and P. V. Ruuskanen, Phys. Rev. C 79, 024903 (2009)

[5] P. Huovinen, P. F. Kolb et. al., Phys. Lett. B 503, 58 (2001)

[6] D. Teaney, J. Lauret and E. V. Shuryak, Phys. Rev. Lett. 86, 4783 (2001)

[7] J. Adams et al. [STAR Collaboration], Phys. Rev. Lett. 92, 052302 (2004) A. Adare et al. [PHENIX Collaboration], Phys. Rev. Lett. 98, $162301(2007)$

[8] B. B. Abelev et al. [ALICE Collaboration], JHEP 1506, 190 (2015)

[9] H. Song, S. Bass and U. W. Heinz, Phys. Rev. C 89, 034919 (2014)

[10] B. B. Abelev et al. [ALICE Collaboration], Phys. Rev. C 90, 054901 (2014)

[11] Y. Zhou [ALICE Collaboration], arXiv:1201.4671 [nucl-ex].

[12] Y. Zhou, X. Zhu, P. Li and H. Song, Phys. Rev. C 91, 064908 (2015) 